\title{
Interfacial resistive oxide switch induced by reversible modification of defect structures
}

\author{
S. Tsui, ${ }^{1,2}$ Y. Y. Xue, ${ }^{1}$ N. Das, ${ }^{1}$ Y. Q. Wang, ${ }^{1}$ and C. W. $\mathrm{Chu}^{1,3,4}$ \\ ${ }^{1}$ Department of Physics and Texas Center for Superconductivity, University of Houston, \\ 202 Houston Science Center, Houston, Texas 77204-5002, USA \\ ${ }^{2}$ Department of Physics, California State University-San Marcos, 333 S. Twin Oaks Valley Road, San Marcos, California 92096, USA \\ ${ }^{3}$ Hong Kong University of Science and Technology, Hong Kong, China \\ ${ }^{4}$ Lawrence Berkeley National Laboratory, 1 Cyclotron Road, Berkeley, California 94720, USA
}

(Received 1 April 2009; revised manuscript received 22 September 2009; published 16 October 2009)

\begin{abstract}
We report on electric field induced bipolar resistive switching in metal electrode- $\operatorname{Pr}_{0.7} \mathrm{Ca}_{0.3} \mathrm{MnO}_{3}$ interfaces that exhibit hopping transport. The electrical transport data show that the number of hopping steps needed for a carrier to cross the interfacial layers changes while switching between high and low resistance states. Furthermore, the frequency response of the interfacial ac impedance shows that the layers can be modeled as a percolation network with an effective resistance that is dominated by its bottleneck section. We therefore propose that a reversible creation/annihilation of a few hopping sites within this bottleneck serves as the switching mechanism, which may be of use in the design of future nonvolatile memory devices.
\end{abstract}

DOI: 10.1103/PhysRevB.80.165415

PACS number(s): 73.20.Hb, 68.55.Ln, 77.22.Gm

\section{INTRODUCTION}

Currently, there is great interest in bistable resistance switching across metal electrode-oxide thin-film interfaces, which can be reversibly alternated between a high resistance state (HRS) and a low resistance state (LRS) by electrical pulses of different polarities. ${ }^{1-9}$ In addition to the potential nonvolatile memory applications, the associated mechanisms are of great scientific interest. Although a consensus has been reached that the switching occurs in the interfacial layer and is unlikely to be a purely electronic process, i.e., simple charge trapping/detrapping, debate over the physical origin continues. In the case of $\mathrm{SrTiO}_{3}$, the electromigration of preexisting point defects along crystalline dislocations and the associated changes in the doping level were proposed to be the underlying mechanism. ${ }^{2,6}$ Similar models have also been proposed in more complicated systems such as $\mathrm{Pr}_{0.7} \mathrm{Ca}_{0.3} \mathrm{MnO}_{3-\delta}$ (PCMO), where the electric field $(E)$-induced change in oxygen stoichiometry through longrange electromigration (e.g., on the order of $100 \mathrm{~nm}$ ) is regarded to be the dominant factor, ${ }^{10}$ despite the system being near its optimal doping level. ${ }^{8,9}$ In the present study, we investigate the resistive switching behavior of metal-PCMO thin-film interfaces and propose that the phenomenon stems from another mechanism in disagreement with the popular models proposed thus far for this system.

First, the reported fast switching [within $\Delta t \leq 10^{-7} \mathrm{~s}$ (Refs. 9 and 11)] and the nonvolatile retention (with a decay time $\tau>10^{8} \mathrm{~s}$ at room temperature ${ }^{9}$ ) for PCMO switches present conceptual challenges if one abides by an electromigration framework. The proposed electromigration is related to thermal diffusion, which determines the nonvolatile retention, through the Nernst-Einstein relation. It has been demonstrated that the ratio $\tau / \Delta t$ during an electromigration/ thermal-relaxation process is insensitive to both the diffusion constant and the thickness of the interfacial layer, and is many orders of magnitude smaller than the ratio that has been experimentally observed. ${ }^{12}$ Across the effective thickness $l=V_{\mathrm{p}} / E$, where $V_{\mathrm{p}}$ is the pulsed voltage drop across the switching interface, the electromigration flux-density $J_{\mathrm{M}}$ would only be $|z| q E D n / k T<10^{17} / \mathrm{s} \mathrm{cm}^{2}$, with an average migration distance far shorter than $1 \AA$ using exaggerated values of the diffusion constant $D=5 \times 10^{-14} \mathrm{~cm}^{2} / \mathrm{s}$, ${ }^{9}$ the field $E=10^{6} \mathrm{~V} / \mathrm{cm}$, the effective defect charge $|z|=4$, and the defect density $n=1 /$ cell, where $q$ is electron charge. The relaxation flux density $J_{\mathrm{R}}$, however, is still $D \frac{\Delta n}{\Delta x}$ $\approx D \Delta n E / V_{\mathrm{p}} \gg 10^{10} / \mathrm{s} \mathrm{cm}^{2}$ even with a small $n$ difference $\Delta n=0.01 /$ cell and a large $V_{\mathrm{p}}=100 \mathrm{~V}$, i.e., a $\tau / \Delta t \gg 10^{7}$ is difficult to reach in this framework. It is interesting to note that the ratio $J_{\mathrm{M}} / J_{\mathrm{R}} \leq 10^{7}$ is independent of $D, E$, and even the layer thickness under a fixed $V_{\mathrm{p}}$, but far from the $\tau / \Delta t$ $>10^{15}$ observed. While the migration of point defects may certainly play a role in switches observed using slow, quasistatic voltage sweeps, ${ }^{6,12}$ it is unlikely to be the mechanism for the sub- $\mu$ s switching reported. ${ }^{1-5,9,12}$

Second, it is unclear how the resistance can be alternated in the interfacial layers of PCMO through an electric field induced doping alone. The expected interfacial layer resistivity may reach $10^{5} \Omega \mathrm{cm}$ or higher in its HRS, which is much higher than those of the oxygen-deficient manganites. ${ }^{3}$ The doubts are further deepened by the same temperature dependence in HRS and LRS and high nonlinearity of the resistance, ${ }^{4}$ both of which are different from the observed doping evolution of manganite resistivity. ${ }^{13}$

We therefore investigated PCMO resistive switching by examining the carrier transport and the dielectric spectrum. Three types of carrier transport across the metal-oxide switches have been observed previously: Schottky-type behavior; ${ }^{14,15}$ space-charge-limited conduction (SCLC) (Refs. 4, 11, and 12); and carrier hopping, the latter of which is typically associated with heavy disorder and a large interfacial capacitance. ${ }^{5}$ The sample characteristics seem to vary significantly not only between different materials but also between different electrodes on the same sample surface due to inherent inhomogeneity at these electrode-thin-film interfaces. Broad sample-to-sample variation is simply a result of the poor control of such layers. While defect creation plays a key role in all three cases, they represent different mesostruc- 
TABLE I. The hopping steps $m$ contained in the current path and the associated switching ratios $\left(R_{\mathrm{HRS}} / R_{\mathrm{LRS}}\right)$ between HRS and LRS. $V_{\text {th }}$ is the threshold pulse voltage across the interfacial layer, which is needed to cause switching. Sample D is only marginally switchable, but listed here for comparison. Differences between samples are likely due to inhomogeneities at the electrode-thin-film interface. However, it is clear that $m$ increases as the samples are switched to LRS.

\begin{tabular}{|c|c|c|c|c|c|}
\hline Sample & & A & B & $\mathrm{C}$ & $\mathrm{D}$ \\
\hline Electrode & & $\mathrm{Au}$ & $\mathrm{Al}$ & $\mathrm{Ag}$ & $\mathrm{Ag}$ \\
\hline \multirow[t]{2}{*}{$m$} & LRS & 6 & 40 & 38 & 15 \\
\hline & HRS & 4 & 4 & 21 & 9 \\
\hline$R_{\mathrm{HRS}} / R_{\mathrm{LRS}}$ & & 2.5 & 5 & 2.1 & 1.2 \\
\hline$V_{\mathrm{th}}(\mathrm{V})$ & & 5.2 & 10 & 23 & 52 \\
\hline
\end{tabular}

tures. We concentrate here on the hopping-type PCMO thinfilm samples. ${ }^{5}$ In the following discussion, we will analyze the transport data within the framework of a percolation model at the interface. This model is then used to extract the nature of the carrier hopping range and the associated number of hopping sites. Our results suggest that the switching takes place due to a reversible "soft breakdown" at these sites.

\section{EXPERIMENTAL SETUP}

Epitaxial PCMO films were synthesized by ac sputtering on $\mathrm{LaAlO}_{3}$ substrates at $760{ }^{\circ} \mathrm{C}$ in an $\mathrm{Ar}: \mathrm{O}_{2}=2: 3$ mixed atmosphere at 140 mTorr. Metal electrodes consisting of either $\mathrm{Ag}, \mathrm{Au}$, or $\mathrm{Al}$, and measuring $0.1 \times 2 \mathrm{~mm}^{2}$ were then $\mathrm{dc}$ sputtered onto the films ex situ. The switching pulses were produced by a Keithley 2400 sourcemeter gated by a DEI PVX-4150 function generator. A HP 54502A digital oscilloscope was used to measure the amplitudes and widths of the produced switching pulses, which were typically 5-50 V and $1 \mu \mathrm{s}$, respectively. The dc resistances were measured by a HP 34401A multimeter whereas the ac impedance was obtained via a Solartron SI 1260 analyzer. In order to characterize the interface without interference from the sample bulk, a 3-leads technique was adopted for all measurements. ${ }^{3}$

\section{CONDUCTANCE MODEL}

We have previously argued that the carrier hopping can be distinguished from both the SCLC and Schottky barriers transport models by (i) the polarity independence of the differential admittance $1 / R_{\mathrm{ac}}+\boldsymbol{i} \omega C$, where $\omega$ is the angular frequency, $R_{\mathrm{ac}}$ is the ac resistance, and $C$ is the capacitance; (ii) an unusually large $C$; (iii) a $e^{-\left(T_{0} / T\right)^{\gamma}}$ temperature dependency of $1 / R_{\mathrm{ac}}$, where $T, T_{0}$, and $\gamma$ are the temperature, characteristic temperature, and dimensionality parameter, respectively; and (iv) the exponential $E$ dependency of $1 / R_{\mathrm{ac}}{ }^{5} \mathrm{Sev}-$ eral samples, therefore, were selected based on the hopping transport criteria (listed in Table I).

A single switching interface may be modeled as a sandwich consisting of the metal electrode, the interfacial layer, and the bulk PCMO. The hot carriers will be injected into the interfacial layer from different sides (i.e., the electrode or the bulk) under different polarities. The conductance is expected to be asymmetric for individual Schottky-type electrode-
PCMO interfaces, which may be observed experimentally when using a 3-leads technique. ${ }^{14}$ This could be rather different from the apparent $R_{\mathrm{ac}}$ measured across metal electrode-PCMO-metal electrode sandwiches, in which the presence of two interfaces would effectively create back-toback Schottky junctions that would lead to an apparent symmetric $I-V$ character. Furthermore, an asymmetric $1 / R_{\mathrm{ac}}$ vs $V_{\mathrm{dc}}$ might even be expected under the SCLC mechanism if there is asymmetry of the crystalline point defect distribution between the two sides of the interfacial layers.

We proceed to investigate the conductance behavior of a sample whose switched states, after initial cycling, were obtained by alternating polarities of single $5.2 \mathrm{~V}$ pulses of $1 \mu \mathrm{s}$ width. The observed conductance $1 / R_{\mathrm{ac}}$ at $1 \mathrm{kHz}$, however, appears to be symmetric in nature, i.e., independent of the dc bias polarity in all cases here [Fig. 1(a)], and the $e^{-\left(T_{0} / T\right)^{\gamma}}$-like $T$ dependency of the $1 / R_{\mathrm{ac}}$ exhibits a parameter value $0.6<\gamma<0.9$. This suggests that carrier hopping is the dominant transport mechanism, not Schottky-type junctions. ${ }^{5}$ To further distinguish from the SCLC case, the $1 / R_{\mathrm{ac}}$ vs $V_{\mathrm{dc}}$ of both the HRS and LRS is plotted in both semilogarithmic scale and logarithmic scale [Figs. 1(b) and 1(c)]. The data are expected to be straight lines for hopping for the former scale and SCLC for the latter scale, respectively. ${ }^{5}$ An exponential $V_{\mathrm{dc}}$ dependency appears again as the best description [Fig. 1(b)]. The slight deviations below $0.05 \mathrm{~V}$ can be attributed to the inhomogeneity in the contact potentials. The SCLC model significantly deviates from the data at a far broader $V_{\text {dc }}$ range of $<0.15 \mathrm{~V}$ [Fig. 1(c)], suggesting it to be a less likely transport interpretation. It is also interesting to note the $E$ dependency at $1 \mathrm{MHz}$ [stars in Fig. 1(b)]. Not only is the detailed functional dependence different, but the $1 / R_{\mathrm{ac}}$ even increases with $V_{\mathrm{dc}}$. As will be discussed later, the high and low frequency data characterize the percolation network and its bottleneck section, respectively, in the carrier hopping model.

We obtained the ac admittance $1 / R_{\mathrm{ac}}+\boldsymbol{i} \omega C$ at $1 \mathrm{kHz}$ as well as the quasistatic differential resistance $d V_{\mathrm{dc}} / d I_{\mathrm{dc}}$ across the interfacial layer, where $V_{\mathrm{dc}}$ and $I_{\mathrm{dc}}$ are the dc bias and the current, respectively, superimposed over the ac excitation. ${ }^{3}$ The $1 \mathrm{kHz} R_{\mathrm{ac}}$ and the quasistatic $d V_{\mathrm{dc}} / d I_{\mathrm{dc}}$ are in good agreement (Fig. 1), and the difference between $1 / R_{\mathrm{ac}}$ at 1 $\mathrm{kHz}$ and $d V_{\mathrm{dc}} / d I_{\mathrm{dc}}$ is therefore ignored in the following discussions. 

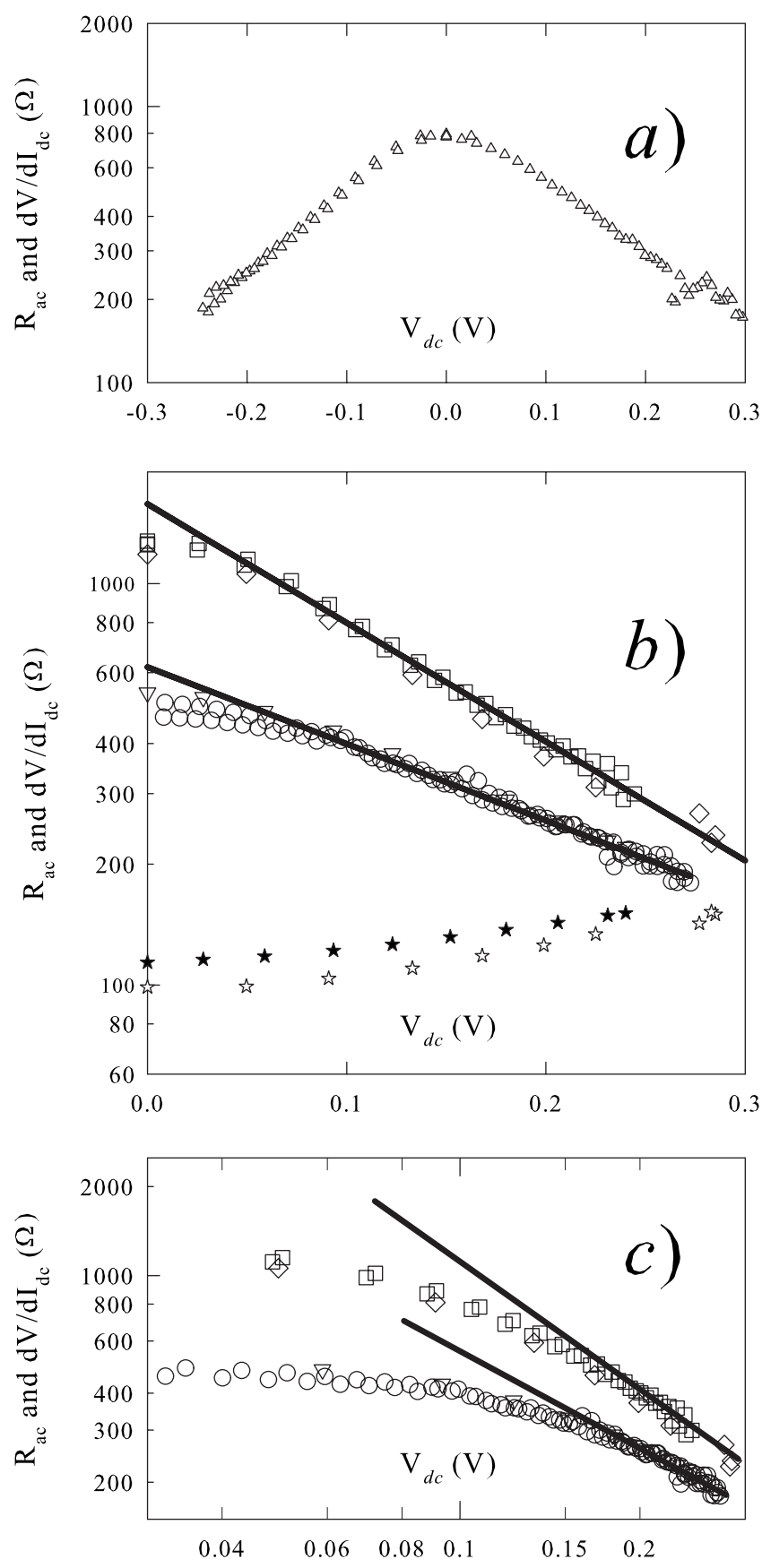

FIG. 1. The differential resistances $d V_{\mathrm{dc}} / d I_{\mathrm{dc}}$ and $R_{\mathrm{ac}}$ under a dc bias $V_{\mathrm{dc}}$ for Sample A. (a) The polarity independence characterizes the hopping transport of the system (here taken at HRS). (b) Semilog plot of the impedance with thick lines fitting the expected exponential behavior for hopping transport. Squares: $d V_{\mathrm{dc}} / d I_{\mathrm{dc}}$ at HRS; diamonds: $R_{\mathrm{ac}}(1 \mathrm{kHz})$ at HRS; circles: $d V_{\mathrm{dc}} / d I_{\mathrm{dc}}$ at LRS; triangles: $R_{\mathrm{ac}}(1 \mathrm{kHz})$ at LRS and; stars: $R_{\mathrm{ac}}(1 \mathrm{MHz})$ at both HRS and LRS. (c) Log-log plot of the impedance with thick lines showing the fit expected of SCLC; clearly this is not preferred over hopping.

\section{REVERSIBLY RECOVERED HOPPING SITES}

In light of the hopping characteristic, it would not be unreasonable to suggest that an electric field induced change in the hopping paths would lead to resistance switching. There-

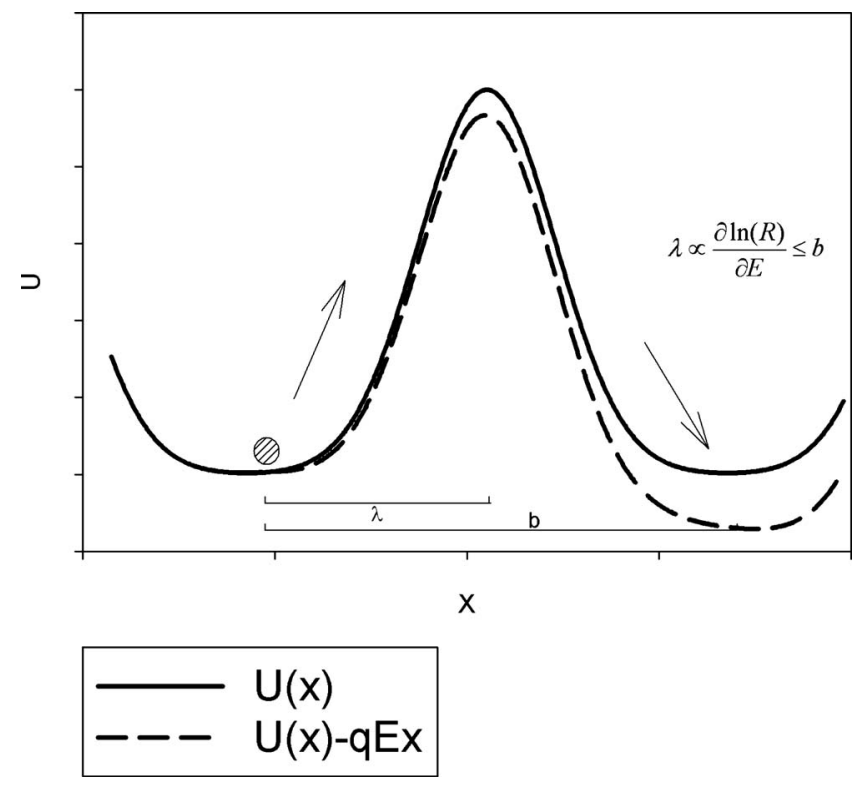

FIG. 2. Schematic representation of the potential barrier associated with a hopping site in the switching interface. The bold line represents the barrier height $U$ to be overcome in order to cross a hopping distance $b$. The dashed line represents the suppressed barrier height in the presence of applied electric field $E$. The displacement $\lambda$ represents the distance between the carrier and the barrier peak. The value of $\lambda$, which is proportional to the slope of the electric field dependence of the resistance, also serves as the lower limit of $b$.

fore, the $E$ dependency of the resistance is further analyzed to extract the relevant information about the carrier hopping ranges. Along hopping paths, a general correlation between the $E$ dependency of $R$ and the hopping distance $b$ (or its lower limit) exists. From this starting point, we can proceed to determine the effective $m$ number of "hops" needed to cross the switching layer. The thermally assisted hopping conductivity actually measures the fraction of carriers with energy higher than the hopping barrier $U$, i.e., $R \propto e^{-U / k T}$, where $k$ is Boltzmann's constant. The effective barrier under an electric field $\mathbf{E}$, however, becomes $U-q \mathbf{E} \cdot \mathbf{x}$, where $\mathbf{x}$ is a general vector along the hopping direction. Therefore, an effective barrier height reduction of $q \mathbf{E} \cdot \boldsymbol{\lambda}$ is expected for the hopping between two fixed sites under applied bias, where $\boldsymbol{\lambda}$ is the actual displacement vector from the hopping origin to the barrier peak (Fig. 2). ${ }^{16}$ Therefore, one would expect $\lambda$ to serve as the lower limit of $b$ and hence be extracted from the derivative of the field dependence, i.e., $-\frac{k T}{q} \cdot \frac{\partial[\ln (R)]}{\partial E}=|\lambda|$. In more complicated variable-range hopping, where the conducting path varies with electric field $E,-\frac{k T}{g} \cdot \frac{\partial[\ln (R)]}{\partial E}=b$ has also been suggested. ${ }^{17}$ The constraint $-\frac{k T}{q} \cdot \frac{\partial[\ln (R)]^{\partial E}}{\partial E} \leq b$, therefore, is a general one. For example, even in the case of Poole-Frenkel field emission, where $R(E) \propto e^{-\sqrt{q^{3} E / \varepsilon^{\prime}} / k T}$ appears rather different from typical hopping, the resulting $\sqrt{q^{3} E / \varepsilon^{\prime}}$ (the energy to the potential maximum) is still the lower limit associated with the carrier travel distance, where $\varepsilon^{\prime}$ is the permittivity. ${ }^{18}$

While characterizing this hopping conduction, it should be pointed out that a major uncertainty arises with the esti- 
mation of the electric field $E=V_{\mathrm{p}} / l$ due to the uncertainty in the effective layer thickness $l$. Although we cannot experimentally determine $l$, this uncertainty can be avoided if one uses the average hopping steps (or its upper limit) $m=l / b$ $=\left(V_{\mathrm{p}} / E\right) / b=-\left[\frac{k T}{q} \cdot \frac{\partial(\ln R)}{\partial V_{\mathrm{p}}}\right]^{-1}$, which can be extracted from the data and mathematically absorbs the value of $l$. It is interesting to note that $m$ is also the ratio between the expected carrier impact energy and the total applied bias potential energy. The energy gain, $q V_{\mathrm{p}}$, of carriers under applied $V_{\mathrm{dc}}$ is mainly absorbed by the $m$ stopping sites. Hence, the average impact energy will be $\Delta K=-q V_{\mathrm{p}} / m=-q V_{\mathrm{p}} \frac{k T}{q} \cdot \frac{\partial(\ln R)}{\partial V_{\mathrm{p}}}=q E \cdot b,{ }^{19}$ which directly determines the defect creation on the one hand and is independent of the $l$ value on the other.

It is rather surprising to find that the $m$ can be extremely small, 4-20 at HRS for most samples examined (Table I). This is the key observation here and has far-reaching implications. First, the minimum impact energy $q V_{\mathrm{th}} / m$ of the carriers needed, where $V_{\text {th }}$ is the threshold voltage required for switching, will be $1 \mathrm{eV}$ or higher for all samples in Table I. The displacement of ions seems to be unavoidable under such impact energy. Second, the number of effective hopping steps $m=l / b$ needed to cross the interfacial layer is different between HRS and LRS (Table I). With $l$ unlikely to be significantly altered (as will be discussed later), the hopping distance $b$ has to be much longer in HRS. Either the corresponding ions are directly displaced, or their surrounding lattices are so distorted by the pulses that a gap opens around the local Fermi level.

Both the small $m$ and the large capacitance observed suggest an $l$ on the order of a few nm. Much thicker interfacial layers, however, have been suggested by scanning Kelvin probe microscopy (SKPM). ${ }^{20}$ To explore the controversy, we analyzed the impedance spectrum, i.e., the response of the switching interfaces with respect to small ac excitations across various frequencies. Strong dispersions are observed in both $R$ and $C$ with respect to frequency [Fig. 3(b)]. Similar dispersions have been shown to be intrinsically related to the switching of the SCLC-type interfacial layers. ${ }^{12}$ As previously demonstrated, ${ }^{21-25}$ mesoscopic heterogeneity is a natural explanation for such frequency responses. Each homogeneous microscopic "domain" along the current path can be represented as a circuit of parallel connected $R_{\mathrm{i}}$ and $C_{\mathrm{i}}$ [inset, Fig. 3(b)], with $R_{\mathrm{i}}=l_{\mathrm{i}} / s_{\mathrm{i}} \sigma_{\mathrm{i}}$ and $C_{\mathrm{i}}=s_{\mathrm{i}} \varepsilon_{\mathrm{i}}^{\prime} \varepsilon_{0} / l_{\mathrm{i}}$, where $s_{\mathrm{i}}, \sigma_{\mathrm{i}}, l_{\mathrm{i}}$, and $\varepsilon_{\mathrm{i}}^{\prime}$ are the cross-sectional area, the conductivity, the length, and the dielectric constant, respectively, of the $i$ th section. All domains along one path and made of the same material and can therefore be simplified as a single RC circuit with a time constant $=\varepsilon_{\mathrm{i}}^{\prime} \varepsilon_{0} \sigma_{\mathrm{i}}$. The inhomogeneity can thus be analyzed as a summation of series-connected RC circuits. ${ }^{21,22} \mathrm{We}$ apply this physical picture to our impedance spectrum data.

\section{TWO-CIRCUIT MODEL}

To evaluate the spectrum quantitatively, we sought to extract the intrinsic interfacial impedance $Z_{0}=R(\omega)-i / \omega C$, keeping in mind the previously mentioned circuit model. However, there are naturally some inhomogeneities across the width of the interface underneath the electrode. To ac-

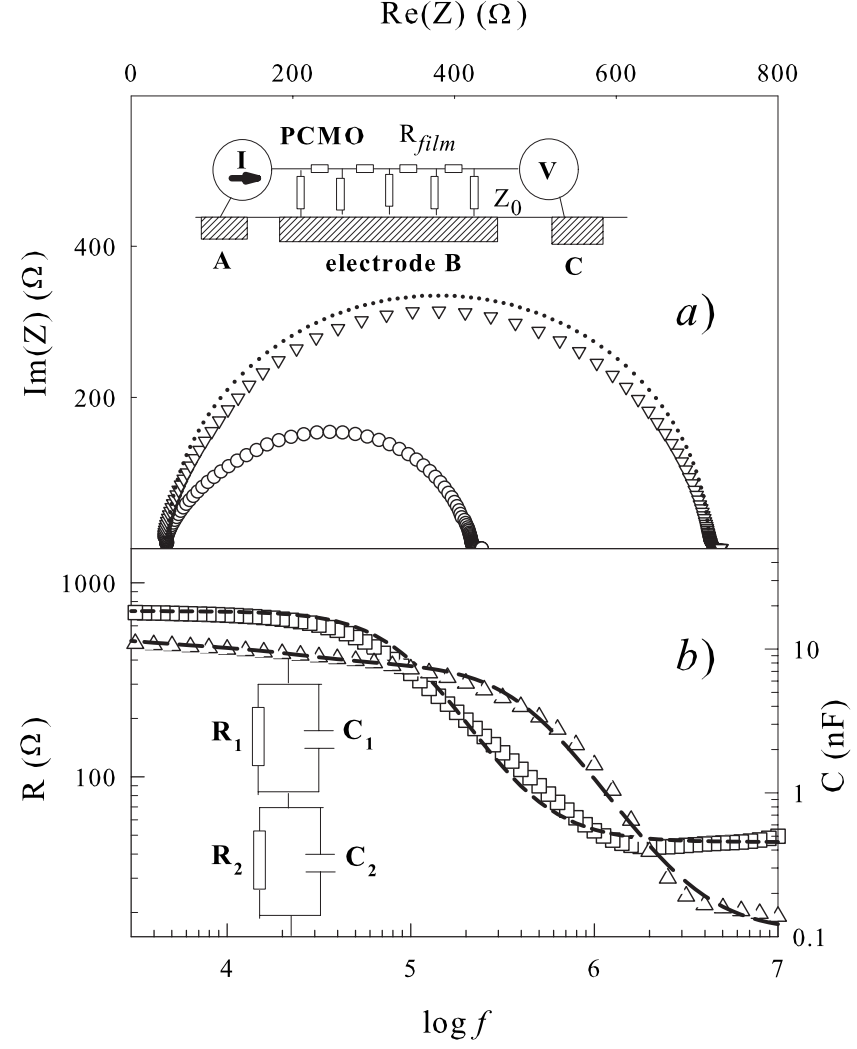

FIG. 3. Impedance data for Sample A. (a) Nyquist plots at HRS (down triangles) and LRS (circles). The dotted line is the HRS fit. Inset: a schematic of the one-dimensional transmission line model used to correct the data above $1 \mathrm{MHz}$. (b) The frequency dependence of the HRS data after correction for the film contribution: $R$ (squares) and $C$ (up triangles). The dashed lines are fits to the twosectioned RC circuit model with $R_{2}$ and $C_{2}$ corresponding to the low frequency plateaus, where the percolative bottleneck dominates. Inset: the two-section model used in the fit.

count for this, we invoked a standard one-dimensional transmission line approximation across the switching electrode [labeled electrode B in the inset of Fig. 3(a)], which yielded only a minor correction to the impedance values above 1 MHz. The Nyquist plot of $-Z^{\prime \prime}$ vs $Z^{\prime}$ [Fig. 3(a)] and complicated dispersions [Fig. 3(b)] of Sample A after such small corrections can be fit rather well with two series-connected parallel sections $R_{1}-C_{1}$ and $R_{2}-C_{2}$ [inset, Fig. 3(b) and Table II], as was expected. The quantities $C_{1} \ll C_{2}$ and the total $R(\omega<100 \mathrm{kHz}) \approx R_{2}$ further demonstrate that the interfacial layer is actually a thin barrier layer (the bottleneck section dominated by $R_{2}$ and $C_{2}$ ) embedded in a much thicker conducting matrix (dominated by $R_{1}$ and $\left.C_{1}\right){ }^{24}$ This model is supported by the very different $E$ dependencies of $R(1 \mathrm{MHz}) \approx R_{1}$ and $R(1 \mathrm{kHz}) \approx R_{2}$ (Fig. 1), and also solves the above thickness controversy: while SKPM likely probes the whole interfacial layer (hence, greater thickness), both $m$ and $C$ measurements examine the thinner dominant bottleneck barrier only (as prescribed by the Maxwell-Wagner mechanism ${ }^{23}$ ). To estimate the permittivity $\varepsilon^{\prime}$, a few virgin $\mathrm{Au}$ electrode-PCMO interfaces were measured. Both the virgin $R(\omega) \approx 50-400 \Omega$ and $C(\omega) \approx 100-200 \mathrm{pF}$ are $\omega$ independent up to $10 \mathrm{MHz}$. This sets an upper limit $\approx 25$ for the 
TABLE II. The parameters of the equivalent two-section RC circuit as determined by the impedance spectra. Note that only $R_{2}$ and $C_{2}$ change upon switching, which suggests that $R_{1}$ and $C_{1}$ represent the conducting percolative matrix, and that $R_{2}$ and $C_{2}$ represent the resistive bottleneck section.

\begin{tabular}{|c|c|c|c|c|c|}
\hline Sample & & A & B & $\mathrm{C}$ & $\mathrm{D}^{\mathrm{a}}$ \\
\hline \multirow[t]{4}{*}{ LRS } & $R_{1}(\Omega)$ & $45 \pm 5$ & 80 & 15 & \\
\hline & $C_{1}(\mathrm{nF})$ & $0.11 \pm 0.02$ & 0.13 & 0.5 & \\
\hline & $R_{2}(\Omega)$ & $375 \pm 10$ & 200 & 630 & 220 \\
\hline & $C_{2}(\mathrm{nF})$ & $10.5 \pm 0.5$ & 1 & 2.8 & 1.5 \\
\hline \multirow[t]{4}{*}{ HRS } & $R_{1}(\Omega)$ & $45 \pm 5$ & 80 & 17 & \\
\hline & $C_{1}(\mathrm{nF})$ & $0.11 \pm 0.02$ & 0.13 & 0.4 & \\
\hline & $R_{2}(\Omega)$ & $670 \pm 20$ & 340 & 780 & 270 \\
\hline & $C_{2}(\mathrm{nF})$ & $9.0 \pm 0.5$ & 0.98 & 2.6 & 1.5 \\
\hline
\end{tabular}

${ }^{a}$ Both $\mathrm{R}$ and $\mathrm{C}$ of Sample D are $\omega$ independent up to $1 \mathrm{MHz}$. The two sections, therefore, cannot be well separated.

$\varepsilon^{\prime}$ by using the film thickness, $300 \mathrm{~nm}$, as the layer thickness. The corresponding $l_{2}$ of the bottleneck is 4 and $16 \mathrm{~nm}$ for $\varepsilon^{\prime}=25$ and 100 , respectively. Consequently, the local field may be as high as $10^{7} \mathrm{~V} / \mathrm{cm}$ with the switching threshold value $V_{\text {th }}=5 \mathrm{~V}$. At such stress, not only is the local ion mobility tremendously enhanced by carrier impact, but also direct $E$-induced displacements/rearrangements are also expected.

Another important feature in Table II is that only $R_{2}$, the hopping resistance across the bottleneck section, changes significantly by switching. The $C_{2}$ variation, however, is within the experimental resolution, confirming the above conjecture that the $l_{2}$ is not significantly affected. The changes in both $C_{1}$ and $R_{1}$ are even smaller. Thus, the switching seems to involve the hopping distances across a thin bottleneck section only.

\section{NONACCUMULATIVE PROCESS}

Because our model contradicts the proposed electromigration model, it is important to address whether the switching process can occur solely due to a fast electric pulse without any prolonged accumulation effects. To exclude the interference of slow processes, such as long-range migration, a series of $\Delta t=1 \mu$ s voltage pulses with monotonically increasing applied amplitude from $V_{\text {app }}=0$ to $+40 \mathrm{~V}$ were applied to a sample. The $R_{\mathrm{ac}}$ was measured after each pulse. A Z-like $V_{\text {app }}$ dependence of the $1 / R_{\mathrm{ac}}$ begins to appear. The pulsing sweep is then reversed and taken to negative polarity. After several pulsed sweep cycles, two stable and repeatable conductance plateaus are established [Fig. 4(a)], which demonstrates the bistable nature of the switching as well as the existence of a threshold voltage $V_{\text {th }}$ required to cross between the $1 / R_{\mathrm{ac}}$ plateaus. As such, a transition between LRS and HRS can be achieved by the application of a single pulse $V_{\text {app }}>V_{\text {th }}$, and the $1 / R_{\text {ac }}$ value of the plateau will be only moderately affected by the pulsing or sweep history. Figure 4(b), for example, shows an early sweep cycle from Sample A that exhibits a down-shifted LRS conductance plateau in the negative branch, which was measured after performing the positive sweep. This is not uncommon, since continued
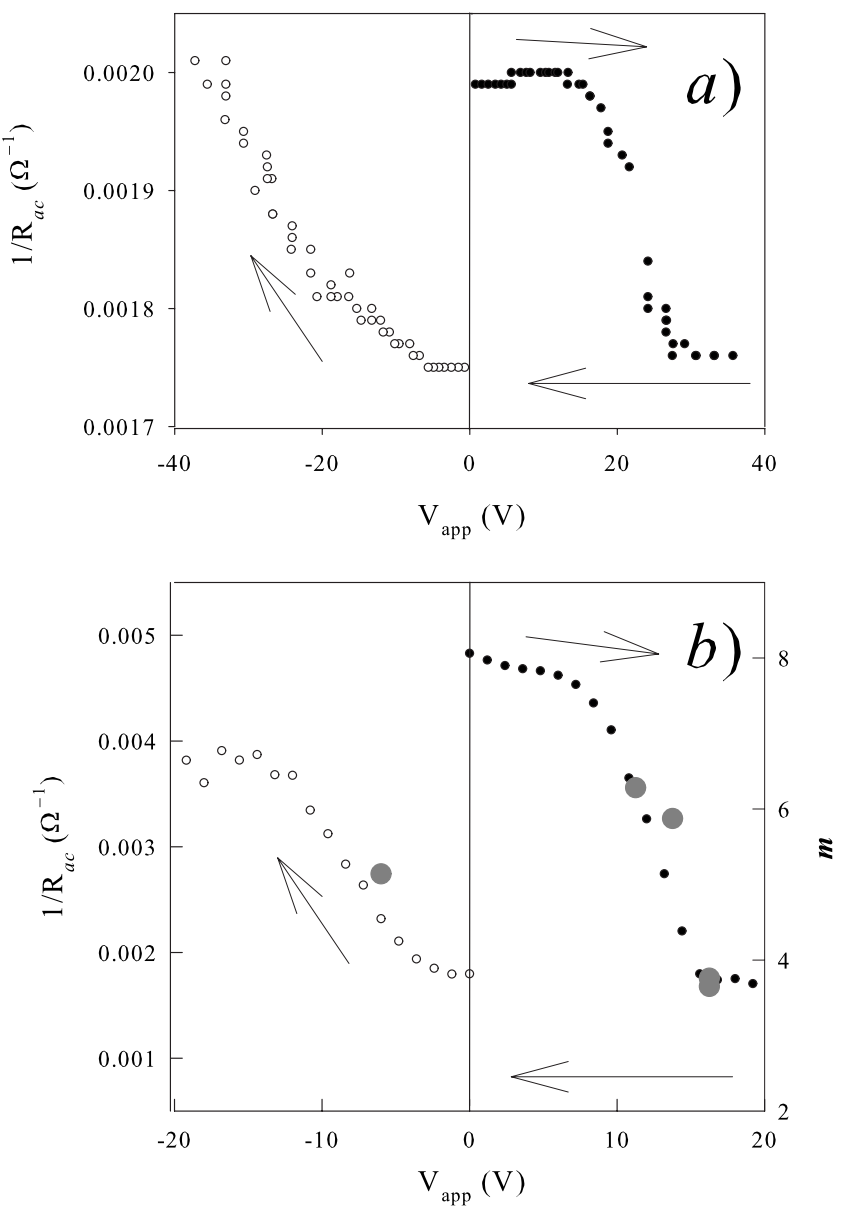

FIG. 4. Conductance values after applied voltage pulsing for various cycles (black symbols). (a) The $1 / R_{\mathrm{ac}}$ for a sample after continuous cycling at various total applied voltages pulses $V_{\text {app }}$. Two bistable conductance plateaus are well established. The arrows indicate the pulsing sequence. Note that neither an accumulative nor electromigration behavior is present. (b) The $1 / R_{\mathrm{ac}}$ during an early sweep for a young Sample A. The arrows indicate the pulsing sequence. The difference in the $1 / R_{\mathrm{ac}}$ at LRS between the two branches might be a result of relaxation which is not uncommon for samples that have undergone relatively few pulsing cycles. However, the $m$ number (gray dots) of hopping sites remains well correlated with the various conductance values. 
cycling tends to increase the resistance. Eventually, repeated cycling will "train" the sample into possessing even conductance plateau values between the positive and negative branches, as is evident in the more established bistable behavior of the sample in Fig. 4(a). However, it is especially interesting to note that the extracted $m$ values not only repeat characteristic values at both LRS and HRS after the training process (Table I), but vary proportionally with the simultaneously measured $1 / R_{\mathrm{ac}}$ even in the early (typically "nonstable") cycles [Fig. 4(b)]. This strongly supports our model that the annihilation/recovery of hopping steps is the mechanism for the bistable switching. It should be cautioned that reversible (partial) switching may also occur far below $\left|V_{\mathrm{th}}\right|$ with smaller $\Delta R=R_{\mathrm{HRS}}-R_{\mathrm{LRS}}$ if much longer electric pulses (or even a quasistatic voltage sweep) are applied. From our observations, these partial switch states induced by subthreshold voltages are rather metastable and typically relax to a higher resistive value. As discussed elsewhere, ${ }^{26}$ the two cases represent two different mechanisms, and electromigration plays important roles only in such subthreshold switching. Also, both the $R_{\mathrm{ac}}$ value and the $V_{\mathrm{p}}$ dependency vary broadly from sample to sample: $V_{\text {th }}$ as low as $1 \mathrm{~V}$ has been observed and $\Delta R$ sometimes even appears as "multilevel switching" with $V_{\text {app. }}{ }^{2}$

\section{DISCUSSION}

The hopping site model for switching may solve two puzzles previously discussed, i.e., the experimentally observed contradictions to models supporting either electromigration or doping. First, the eV-level impact energy is several orders of magnitude larger than the thermal energy. The switching speed $\Delta t$, which is associated with the impact energy, and the retention time $\tau$, which should be associated with the thermal energy, may belong to totally different processes and no longer be constrained by the Nernst-Einstein correlation. Second, the change in $m$ offers a natural way to understand the dilemma that the apparent hopping activation energy of the low-frequency $R_{\mathrm{ac}}$ for different resistance states is not affected by switching. ${ }^{4}$ The experimentally deduced hopping activation energy $k \cdot \frac{\partial[\ln (R)]}{\partial(1 / T)}$, for example, changes only $0.01 \mathrm{eV}$ between HRS and LRS for Sample A despite the twofold $R$ increase observed. This is in disagreement with models invoking either doping ${ }^{9}$ or metal-insulator transition. ${ }^{7}$ For quasi-one-dimensional hopping, missed hopping sites may modify the wave-function overlap, and therefore $R$, without affecting the barrier height. ${ }^{27}$

Similar defect creations in $\mathrm{SiO}_{2}$ by electric fields are well documented. ${ }^{28}$ The associated percolation current through filamentary paths, $\approx 1-100 \mathrm{~nm}^{2}$ in cross-section, the exponential $I-V$ characteristics, and even the spontaneous $R$ switching/jumps are rather similar to those observed here. ${ }^{28,29}$ These defect creations, however, are unrecoverable, polarity independent, and accumulative. Whether electric field created lattice defects can be healed under the opposite field, therefore, is a major issue that needs to be addressed.

While the detailed physics of such recoverability is still unclear, we offer some tentative comments. First, the ionic nature of PCMO may be important. The ionic bond is essentially a potential minimum of the combined long-range Coulomb attraction and hard-core repulsions. No additional barriers associated with the electron orbital reconfigurations exist. Rearrangement of charged ions can therefore be very fast, as demonstrated in Ref. 30. Second, the proper mesostructures with highly localized fields may be crucial. The recoverable hopping sites may involve only a few ions clustered within a bottleneck length scale of $l_{2} / m<1 \mathrm{~nm}$, where both the field and carrier impacts are concentrated. Such small dimensions may favor reversible and polaritydependent ion rearrangements under carrier impact, i.e., only a few states may be stable enough to survive reversible reconfiguration and thermal relaxations. Third, such small structures, where the resistance of the network limits the local switching current, may be preferred for this phenomenon. We therefore suggest that a reversible and bistable "soft breakdown" may occur in ionic crystals with proper mesostructures, although the details require further investigation.

\section{CONCLUSION}

In summary, we show that the electrode-PCMO interfacial layer with dense defects can be modeled as a percolation network with a nm-scale bottleneck section, across which the effective carrier hopping range determines the resistance. An $m$ number of sites along these key hopping paths can be reversibly displaced/healed by pulses with opposing polarities, which yields the bipolar resistive switch. The switching, therefore, is essentially realized at the atomic level. An engineering challenge to the field would be the controlled fabrication and manipulation of such mesostructures, which presently are observed in rather inhomogeneous interfaces. A recoverable soft breakdown stage is thus proposed for ionic crystals with the proper mesostructures, which may form the basis for nano-memory devices if confirmed by further experiments.

\section{ACKNOWLEDGMENTS}

The authors would like to thank J. Shulman for his insightful comments. This work is supported in part by the T. L. L. Temple Foundation, the John J. and Rebecca Moores Endowment, the State of Texas through the Texas Center for Superconductivity, the U.S. Air Force Office of Scientific Research, and at Lawrence Berkeley Laboratory by the Director, Office of Science, Office of Basic Energy Sciences, Division of Materials Sciences and Engineering of the U.S. Department of Energy under Contract No. DE-AC0376SF00098. 
${ }^{1}$ S. Q. Liu, N. J. Wu, and A. Ignatiev, Appl. Phys. Lett. 76, 2749 (2000).

${ }^{2}$ A. Beck, J. G. Bednorz, Ch. Gerber, C. Rossel, and D. Widmer, Appl. Phys. Lett. 77, 139 (2000).

${ }^{3}$ A. Baikalov, Y. Q. Wang, B. Shen, B. Lorenz, S. Tsui, Y. Y. Sun, Y. Y. Xue, and C. W. Chu, Appl. Phys. Lett. 83, 957 (2003).

${ }^{4}$ S. Tsui, A. Baikalov, J. Cmaidalka, Y. Y. Sun, Y. Q. Wang, Y. Y. Xue, C. W. Chu, L. Chen, and A. J. Jacobson, Appl. Phys. Lett. 85, 317 (2004).

${ }^{5}$ S. Tsui, Y. Q. Wang, Y. Y. Xue, and C. W. Chu, Appl. Phys. Lett. 89, 123502 (2006).

${ }^{6}$ K. Szot, W. Speier, G. Bihlmayer, and R. Waser, Nature Mater. 5, 312 (2006).

${ }^{7}$ M. J. Rozenberg, I. H. Inoue, and M. J. Sanchez, Phys. Rev. Lett. 92, 178302 (2004).

${ }^{8}$ M. Quintero, P. Levy, A. G. Leyva, and M. J. Rozenberg, Phys. Rev. Lett. 98, 116601 (2007).

${ }^{9}$ Y. B. Nian, J. Strozier, N. J. Wu, X. Chen, and A. Ignatiev, Phys. Rev. Lett. 98, 146403 (2007).

${ }^{10}$ See, for example, R. Meyer, R. Waser, J. Helmbold, and G. Borchardt, Phys. Rev. Lett. 90, 105901 (2003).

${ }^{11}$ See, for example, A. Odagawa, H. Sato, I. H. Inoue, H. Akoh, M. Kawasaki, Y. Tokura, T. Kanno, and H. Adachi, Phys. Rev. B 70, 224403 (2004).

${ }^{12}$ N. Das, S. Tsui, Y. Y. Xue, Y. Q. Wang, and C. W. Chu, Phys. Rev. B 78, 235418 (2008).

${ }^{13}$ A. P. Ramirez, J. Phys.: Condens. Matter 9, 8171 (1997).

${ }^{14}$ A. Sawa, T. Fujii, M. Kawasaki, and Y. Tokura, Appl. Phys. Lett. 85, 4073 (2004).

${ }^{15}$ T. Fujii, M. Kawasaki, A. Sawa, Y. Kawazoe, H. Akoh, and Y. Tokura, Phys. Rev. B 75, 165101 (2007).
${ }^{16}$ D. H. Dunlap, P. E. Parris, and V. M. Kenkre, Phys. Rev. Lett. 77, 542 (1996).

${ }^{17}$ B. Sanjai, A. Raghunathan, T. S. Natarajan, G. Rangarajan, S. Thomas, P. V. Prabhakaran, and S. Venkatachalam, Phys. Rev. B 55, 10734 (1997).

${ }^{18}$ J. Frenkel, Phys. Rev. 54, 647 (1938).

${ }^{19}$ See, for example, Y. Okuto and C. R. Crowell, Phys. Rev. B 6, 3076 (1972).

${ }^{20}$ X. Chen, N. Wu, J. Strozier, and A. Ignatiev, Appl. Phys. Lett. 89, 063507 (2006).

${ }^{21}$ See, for example, D. C. Sinclair, T. B. Adams, F. D. Morrison, and A. R. West, Appl. Phys. Lett. 80, 2153 (2002).

${ }^{22}$ D. P. Almond and C. R. Bowen, Phys. Rev. Lett. 92, 157601 (2004).

${ }^{23}$ P. Lunkenheimer, V. Bobnar, A. V. Pronin, A. I. Ritus, A. A. Volkov, and A. Loidl, Phys. Rev. B 66, 052105 (2002).

${ }^{24}$ See, for example, J. C. Dyre and T. B. Schrøder, Rev. Mod. Phys. 72, 873 (2000).

${ }^{25}$ See, for example, J. T. S. Irvine, D. C. Sinclair, and A. R. West, Adv. Mater. (Weinheim, Ger.) 2, 132 (1990).

${ }^{26}$ N. Das, S. Tsui, Y. Y. Xue, Y. Q. Wang, and C. W. Chu, Phys. Rev. B 80, 115411 (2009).

${ }^{27}$ See, for example, V. I. Kozub and A. A. Zuzin, Phys. Rev. B 69, 115306 (2004).

${ }^{28}$ See, for example, J. H. Stathis, IBM J. Res. Dev. 46, 265 (2002).

${ }^{29}$ See, for example, T. P. Chen, M. S. Tse, X. Zeng, and S. Fung, Semicond. Sci. Technol. 16, 793 (2001).

${ }^{30}$ See, for example, M. E. Leunissen, C. G. Christova, A. Hynninen, C. P. Royall, A. I. Campbell, A. Imhof, M. Dijkstra, R. van Roij, and A. van Blaaderen, Nature (London) 437, 235 (2005). 